\title{
Dual-use: Helpful or harmful?
}

\author{
Anusha Divvi ${ }^{1}$, Shivashankar Kengadaran ${ }^{1}$
}

AFFILIATION

1 Indira Gandhi Institute of Dental Sciences, Puducherry, India

CORRESPONDENCE TO

Anusha Divvi. Indira Gandhi Institute of Dental Sciences, Pillayarkuppam, Puducherry 607402, India. E-mail: anushadivvi1991@gmail.com ORCID ID: https://orcid.org/0000-0001-7024-4594
KEYWORDS

vaping, dual-use, tobacco cessation

Received: 1 December 2019, Revised: 4 December 2019, Accepted: 4 December 2019

Popul. Med. 2019;1(December):6

https://doi.org/10.18332/popmed/115037

\section{DEAR EDITOR,}

Mix use of electronic and combustible cigarettes, referred to as 'dual-use', is perceived as a win by those who are working towards quitting smoking, while others observe it as an absolute health hazard. The latest data from the Population Assessment of Tobacco and Health (PATH) study confirms that dual-users are exposed to higher levels of nicotine and other carcinogens than people who exclusively smoke cigarettes ${ }^{1}$.

An ongoing study among 46000 Americans stated that 792 dual-users were exposed to the highest concentrations of toxic substances compared with 247 people who only vaped and 2411 who only smoked ${ }^{1}$. People who exclusively use electronic cigarettes were exposed to the lowest amounts of lethal substances compared to exclusive smokers and dualusers. Yet, there is ambiguity in the conclusion that dual-use is a dangerous option than smoking alone ${ }^{1}$.

A study on retrospective PATH data stated that cigarette smoking frequency could be a reason for high concentrations of toxicants among dual-users. Out of 982 dual-users in that study, $82 \%$ reported daily cigarette smoking, which was positively correlated with the presence of carcinogens ${ }^{2}$.

It was also hypothesized that, among dual-users, the additional nicotine from electronic cigarettes may replace nicotine levels of smoking traditional cigarettes and thereby decrease cigarette consumption ${ }^{3}$. However, in all actuality, electronic cigarettes deliver lower levels of plasma nicotine in contrast to traditional cigarettes, which could leave dual-users unsatisfied and prone to titrate their nicotine intake ${ }^{4}$. Along these lines, it is unclear whether dual-users are smoking fewer cigarettes per day and lessening their exposure to tobacco smoke and nicotine, or they are smoking to the same extent and merely introducing more toxicants and nicotine from the additional use of electronic cigarettes ${ }^{5}$.

A cross-sectional analysis of electronic cigarette use, among 449092 never and current traditional cigarette smokers, found that the odds of cardiovascular disease were $36 \%$ higher among dual-users in comparison with current traditional cigarette smokers who never used electronic cigarettes $^{6}$. Therefore, dual-use is more dangerous than smoking alone, and has to be considered as a major public health concern.

Given that FDA-approved drugs for tobacco treatment in combination with behavioural counselling remain the most efficient means of tobacco cessation, it appears that there is an earnest need to train healthcare providers to develop strong anti-vaping messages in tobacco cessation programs and increase the acceptability of FDA-approved medications among tobacco users. Dual-users should be encouraged to stop smoking cigarettes and electronic cigarettes to reduce health risks. There is also an urgent need for developing more effective tobacco cessation modalities rather than promoting vaping as a safe alternative to smoking.

\section{REFERENCES}

1. United States Department of Health and Human Services, National Institutes of Health, National Institute on Drug Abuse, Food and Drug Administration, Center for Tobacco Products. Population Assessment of Tobacco and Health (PATH) Study [United States] Restricted-Use Files (ICPSR 36231). In: Population Assessment of Tobacco and Health (PATH) Study Series. Published June 3, 2019. Accessed December 1, 2019. doi:10.3886/ICPSR36231.v19

2. Goniewicz ML, Smith DM, Edwards KC, et al. Comparison of nicotine and toxicant exposure in users of electronic cigarettes and combustible cigarettes. JAMA Netw Open. 2018;1(8):e185937. doi:10.1001/jamanetworkopen.2018.5937 3. Wang JB, Olgin JE, Nah G, Vittinghoff E, Cataldo JK, Pletcher MJ, Marcus GM. Cigarette and e-cigarette dual use and risk of cardiopulmonary symptoms in the Health eHeart Study. PLoS ONE. 2018;13(7):e0198681. doi:10.1371/journal.pone.0198681

4. Vansickel AR, Cobb CO, Weaver MF, Eissenberg TE. A clinical laboratory model for evaluating the acute effects of electronic nicotine delivery profile and cardiovascular and subjective effects. Cancer Epidemiol Biomarkers Prev. 2010;19(8):1945- 
1953. doi:10.1158/1055-9965.epi-10-0288

5. Bullen C, McRobbie H, Thornley S, Glover M, Lin R, Laugesen $M$. Effect of an electronic nicotine delivery device (e cigarette) on desire to smoke and withdrawal, user preferences and nicotine delivery: randomised cross-over trial. Tob Control. 2010;19(2):98-103. doi:10.1136/tc.2009.031567

6. Osei AD, Mirbolouk M, Orimoloye OA, et al. The association between e-cigarette use and cardiovascular disease among never and current combustible cigarette smokers: BRFSS 2016 \& 2017. Am J Med. 2019;132(8):949-954. doi:10.1186/s12890-019-0950-3 